\title{
Discovery of a Novel Tetrapeptide against Influenza A Virus: Rational Design, Synthesis, Bioactivity Evaluation and Computational Studies
}

\author{
Maria Carmina Scala ${ }^{1, \dagger}$, Mariangela Agamennone ${ }^{2,+}{ }^{\mathbb{C}}$, Agostina Pietrantoni ${ }^{3,4}$, Veronica Di Sarno ${ }^{1}$,

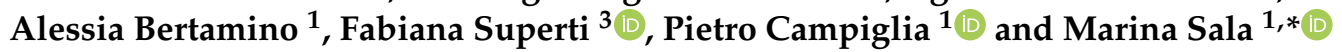 \\ 1 Department of Pharmacy, University of Salerno, Via Giovanni Paolo II 132, 84084 Fisciano, Italy; \\ mscala@unisa.it (M.C.S.); vdisarno@unisa.it (V.D.S.); abertamino@unisa.it (A.B.); pcampiglia@unisa.it (P.C.) \\ 2 Department of Pharmacy, University “G. d'Annunzio" of Chieti-Pescara, Via dei Vestini 31, 66100 Chieti, Italy; \\ magamennone@unich.it \\ 3 National Centre for Innovative Technologies in Public Health, National Institute of Health, Viale Regina Elena \\ 299, 00161 Rome, Italy; agostina.pietrantoni@iss.it (A.P.); fabiana.superti@iss.it (F.S.) \\ 4 Core Facilities, National Institute of Health, Viale Regina Elena 299, 00161 Rome, Italy \\ * Correspondence: msala@unisa.it; Tel.: +39-(0)-89968148 \\ + These authors contributed equally to this work.
}

\section{check for}

updates

Citation: Scala, M.C.; Agamennone, M.; Pietrantoni, A.; Di Sarno, V.; Bertamino, A.; Superti, F.; Campiglia, P.; Sala, M. Discovery of a Novel Tetrapeptide against Influenza A Virus: Rational Design, Synthesis, Bioactivity Evaluation and Computational Studies. Pharmaceuticals 2021, 14, 959. https://doi.org/10.3390/ph14100959

Academic Editor: Zoidis Grigoris

Received: 10 August 2021

Accepted: 20 September 2021

Published: 23 September 2021

Publisher's Note: MDPI stays neutral with regard to jurisdictional claims in published maps and institutional affiliations.

Copyright: (c) 2021 by the authors. Licensee MDPI, Basel, Switzerland. This article is an open access article distributed under the terms and conditions of the Creative Commons Attribution (CC BY) license (https:/ / creativecommons.org/licenses/by/ $4.0 /)$.

\begin{abstract}
Influenza is a highly contagious, acute respiratory illness, which represents one of the main health issues worldwide. Even though some antivirals are available, the alarming increase in virus strains resistant to them highlights the need to find new drugs. Previously, Superti et al. deeply investigated the mechanism of the anti-influenza virus effect of bovine lactoferrin (bLf) and the role of its tryptic fragments (the N- and C-lobes) in antiviral activity. Recently, through a truncation library, we identified the tetrapeptides, Ac-SKHS- $\mathrm{NH}_{2}$ (1) and Ac-SLDC-NH $\mathrm{N}_{2}$ (2), derived from bLf C-lobe fragment 418-429, which were able to bind hemagglutinin (HA) and inhibit cell infection in a concentration range of femto- to picomolar. Starting from these results, in this work, we initiated a systematic SAR study on the peptides mentioned above, through an alanine scanning approach. We carried out binding affinity measurements by microscale thermophoresis (MST) and surface plasmon resonance (SPR), as well as hemagglutination inhibition (HI) and virus neutralization (NT) assays on synthesized peptides. Computational studies were performed to identify possible ligand-HA interactions. Results obtained led to the identification of an interesting peptide endowed with broad anti-influenza activity and able to inhibit viral infection to a greater extent of reference peptide.
\end{abstract}

Keywords: influenza A virus; lactoferrin; tetrapeptides; biophysics; antiviral agents; hemagglutinin

\section{Introduction}

Influenza is a highly contagious, acute respiratory illness, which represents one of the most important health issues worldwide. There are three types of influenza viruses that infect humans: A, B and C. Influenza A viruses (IAV) also naturally infect a variety of other animal species and are the only influenza viruses known to cause influenza pandemics, which are global epidemics of influenza diseases [1]. The recent outbreak of SARS-CoV-2 dramatically demonstrated the risks of a global viral spread and highlighted the key role of prevention. In this context, influenza A virus is a highly monitored pathogen. It is largely diffused in the avian population, and its spillover to humans could represent a serious threat [2]. Despite its global diffusion, just a few drugs are used by clinics, with vaccination representing the main strategy for preventing infections. Efforts to prevent influenza by vaccination are made difficult by the virus's ability to rapidly mutate and recombine into antigenically new viral particles, sometimes leading to the emergence of a totally new viral strain. For this reason, at present, the development of antiviral drugs represents a crucial strategy in the control and prevention of seasonal and pandemic influenza 
infections [3]. Three classes of antiviral drugs have been approved for treatment and prophylaxis of influenza [4]: the adamantane derivatives (amantadine and rimantadine), potent M2 channel blockers [5], neuraminidase inhibitors (NAIs: zanamivir, oseltamivir laninamivir and peramivir) [6,7] and the selective inhibitor of influenza cap-dependent endonuclease (baloxavir marboxil) [8]. However, the capability of viruses to mutate the target proteins represents an obstacle to efficient treatment with these drugs. Based on the above considerations, the need for new compounds against influenza virus able to overcome the disadvantages of the known therapies is evident $[9,10]$.

An attractive antiviral strategy is the blocking of influenza virus entry into the host cell. This process is mediated by viral hemagglutinin. HA is the major surface protein of IAV and is essential to the entry process, thus representing an attractive target for antiviral therapy. It is a large, homotrimeric, mushroom-shaped glycoprotein responsible for initial attachment to the host cell through the receptor-binding site (RBS) and successive viral internalization through membrane fusion promoted by structural rearrangement of the conserved stem region of HA (Figure 1). As a matter of fact, neutralizing compounds targeting HA represents a useful tool in neutralizing viral infection. One notable difficulty in targeting HA is related to its sequence variability among different strains (18 HAs have been identified so far) [11].

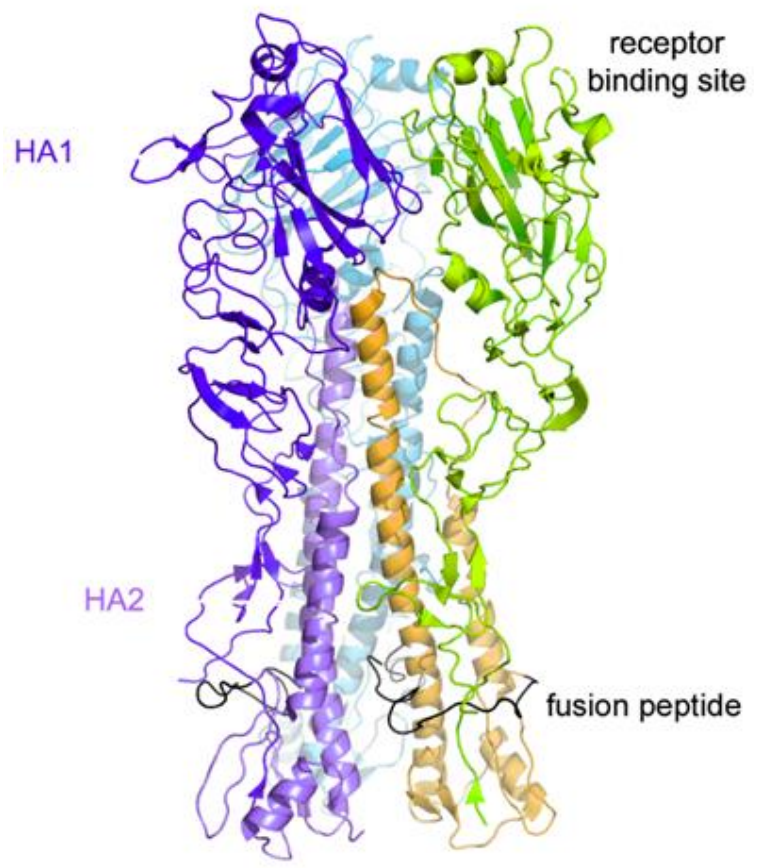

Figure 1. Cartoon representation of the HA trimeric structure.

Previously, Superti et al. deeply investigated the mechanism of the anti-influenza virus effect of bovine lactoferrin (bLf) and the role of its tryptic fragments (the N- and C-lobes) in antiviral activity [12]. In particular, they evaluated the influence of bLf on hemagglutinin-mediated functions [13].

Recently, through a truncation library, we identified the tetrapeptides Ac-SKHS- $\mathrm{NH}_{2}$ (1) and Ac-SLDC-NH $\mathrm{N}_{2}$ (2) derived from bLf C-lobe fragment 418-429, which were able to bind HA and inhibit cell infection [14]. By using this information, we further investigated the role of key residues of both peptides in the interaction with HA. This will allow better defining the influence of the chemical modifications introduced in the peptides on their biological properties in terms of affinity and activity. Direct binding assays, bioactivity profile and computational studies led to the identification of a very potent and broadspectrum tetrapeptide. 


\section{Results and Discussion}

\subsection{Design and Synthesis}

The contribution of the various amino acid residues to peptides $\mathbf{1}$ and $\mathbf{2}$ activity was further established through L-Ala scanning analysis [15]. This approach allows determining the contribution of side chains of each amino acid residue in the interaction with the target molecule, hemagglutinin. This approach resulted in the generation of a panel of eight peptides (peptides 3-10, Table 1).

Table 1. Structure characterization, affinity and activity of peptides $\mathbf{1 - 1 0}$. The HI activity of peptides $\mathbf{1}$ and $\mathbf{2}$ is reported as a reference. ${ }^{\text {a }}$ Scala et al. (2017) [14]. All peptides are amidated and acetylated. ${ }^{\text {a }}$ The HI titer, determined by the hemagglutination inhibition assay, corresponds to the concentration of the peptide that produces $50 \%$ inhibition of virusinduced hemagglutination, i.e., IC50. ${ }^{\mathrm{b}}$ N.D.: not detectable at the maximum tested concentration $\left(12.5 \times 10^{3} \mathrm{nM}\right)$.

\begin{tabular}{|c|c|c|c|c|c|c|}
\hline \multirow[b]{2}{*}{ Pep. } & \multirow[b]{2}{*}{ Seq. } & \multirow[b]{2}{*}{ MST $K_{D}(\mu M)$} & \multirow[b]{2}{*}{ SPR $K_{D}(\mu M)$} & \multicolumn{3}{|c|}{ HI Titer ${ }^{a}(n M)$} \\
\hline & & & & $\begin{array}{c}\text { A/Roma-ISS/02/08 } \\
\text { H1N1 }\end{array}$ & $\begin{array}{c}\text { A/Parma/24/09 } \\
\text { H1N1 }\end{array}$ & $\begin{array}{c}\text { A/Parma/05/06 } \\
\text { H3N2 }\end{array}$ \\
\hline $\mathbf{1}^{\mathrm{a}}$ & SKHS & $7.26 \pm 0.06$ & $4.53 \pm 0.08$ & 0.1 & 1.5 & 12 \\
\hline 3 & AKHS & $3.12 \pm 0.11$ & $2.7 \pm 0.04$ & 0.6 & N.D. ${ }^{b}$ & 12 \\
\hline 4 & SAHS & $0.0082 \pm 0.0001$ & $0.0035 \pm 0.00012$ & $1.8 \times 10^{-6}$ & 0.5 & $2.4 \times 10^{-6}$ \\
\hline 5 & SKAS & $7.01 \pm 0.09$ & $1.03 \pm 0.01$ & $1.8 \times 10^{-6}$ & $2.9 \times 10^{-3}$ & N.D. ${ }^{b}$ \\
\hline 6 & SKHA & $11.4 \pm 0.17$ & $6.75 \pm 0.81$ & $5 \times 10^{-7}$ & N.D. ${ }^{b}$ & N.D. ${ }^{b}$ \\
\hline $2^{a}$ & SLDC & $10.4 \pm 0.23$ & $7.12 \pm 0.26$ & $1.4 \times 10^{-6}$ & 6 & 1.5 \\
\hline 7 & ALDC & $21.2 \pm 0.41$ & $0.0277 \pm 0.0017$ & N.D. ${ }^{b}$ & $9 \times 10^{-7}$ & $3.6 \times 10^{-7}$ \\
\hline 8 & SADC & $6.38 \pm 0.21$ & $2.19 \pm 0.51$ & N.D. ${ }^{b}$ & $2.2 \times 10^{-6}$ & $5 \times 10^{-7}$ \\
\hline 9 & SLAC & $0.0058 \pm 0.0003$ & $2.57 \pm 0.34$ & N.D. ${ }^{b}$ & $5 \times 10^{-7}$ & $9 \times 10^{-7}$ \\
\hline 10 & SLDA & $2.69 \pm 0.09$ & $0.343 \pm 0.019$ & N.D. $b$ & 6.1 & $2.1 \times 10^{-3}$ \\
\hline
\end{tabular}

Peptides were synthesized according to the solid-phase approach using standard Fmoc methodology in a manual reaction vessel (Material and Methods (Section 3)). The peptides' purification was achieved using a preparative RP-HPLC C-18 bonded silica column. The purified peptides were $98 \%$ pure, as determined by analytical RP-HPLC. The correct molecular weight of the peptides was confirmed by mass spectrometry and amino acid analysis (Table S1, Figures S1-S8, Supplementary Materials).

\subsection{Direct Binding Assays}

There are many biophysical methods available to measure the affinity of ligandprotein interactions. Each technique affords a variety of information on the binding specificity, kinetics thermodynamics and stoichiometry, as well as their advantages and disadvantages, which have been extensively analyzed elsewhere [16]. No single biophysical technique is better than another, as they are based on different principles. For this reason, a more satisfactory approach would be to adopt a strategy that uses one or more orthogonal assays that aim to confirm activity on the target. Based on this consideration, we decided to investigate the direct binding of peptides 1, 2 and the alanine scanning peptide library to HA protein by two complementary techniques: microscale thermophoresis (Figure 2) and surface plasmon resonance (Figure 3). 

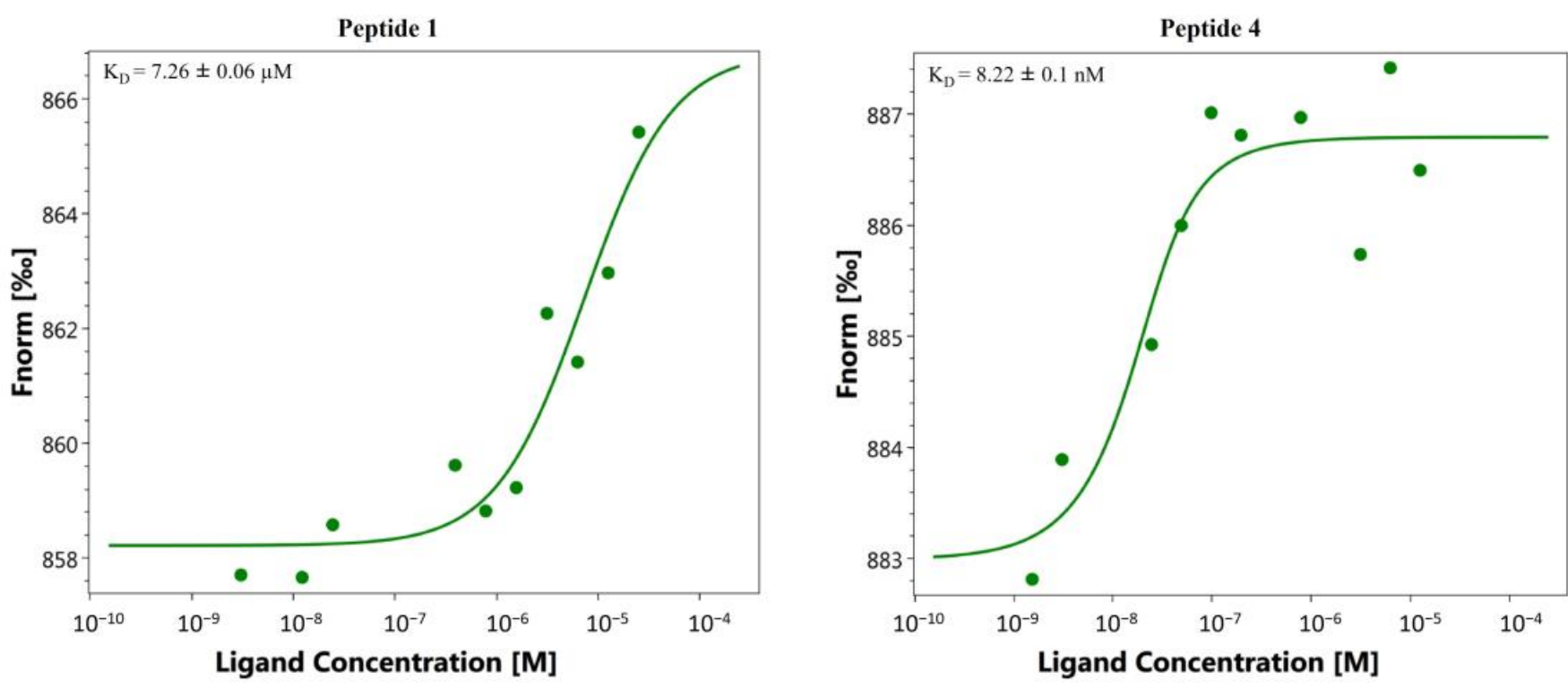

Figure 2. Direct binding measurements of the interaction between HA (H1N1 hemagglutinin, Cat No.: Z03181, GenScript, NE) and peptide 1 and 4. MST binding affinity assays were performed as described in the Materials and Methods section (Section 3). Representative single dose-response curves of peptides 1 and 4 binding to HA are shown; Fnorm, normalized fluorescence. Experiments were repeated independently three times. Reported $\mathrm{K}_{\mathrm{D}}$ is the mean $\pm \mathrm{SD}$ of three independent experiments.
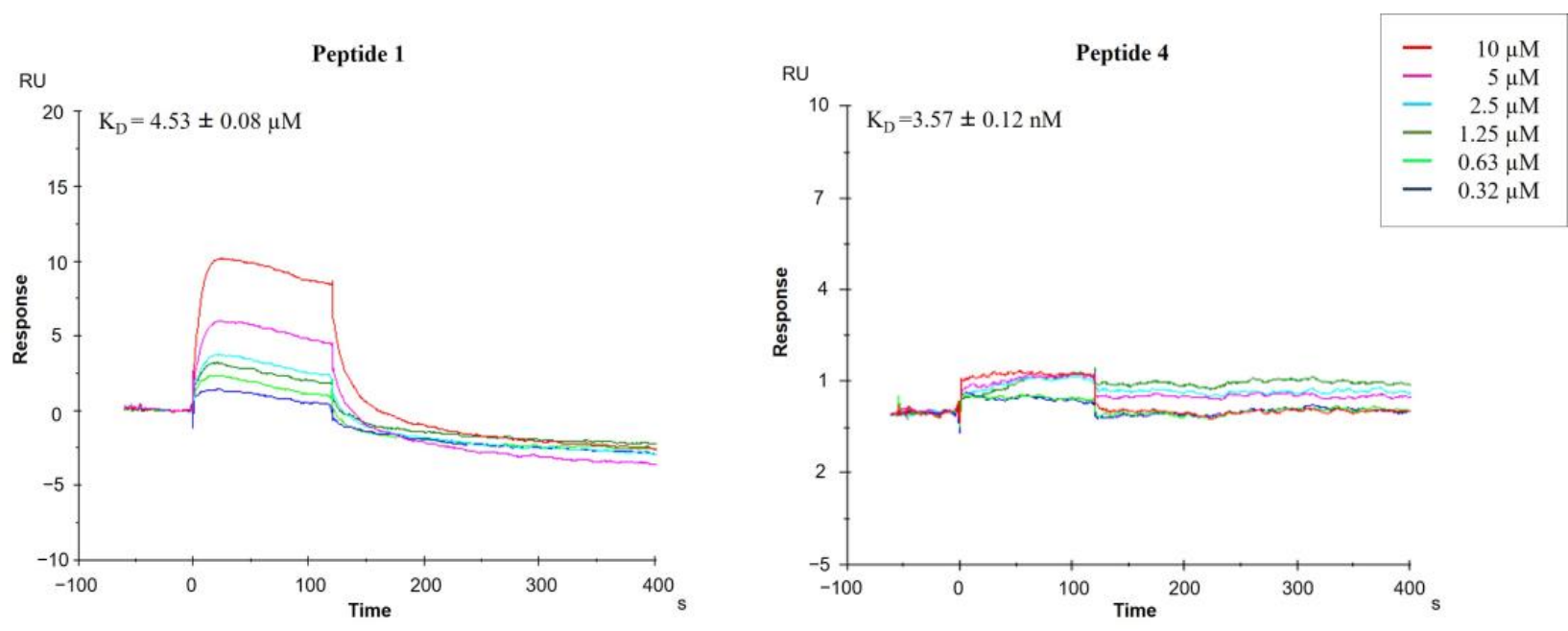

Figure 3. Sensorgrams obtained from the SPR interaction analysis of peptides 1 and 4 binding to immobilized HA (H1N1 hemagglutinin, Cat No: Z03181, GenScript, NE). Each compound was injected at six different concentrations $(0.32,0.63,1.25$, $2.5,5$ and $10 \mu \mathrm{M})$. The equilibrium dissociation constants $\left(\mathrm{K}_{\mathrm{D}}\right)$ were derived from the ratio between kinetic dissociation $\left(\mathrm{k}_{\mathrm{off}}\right)$ and association $\left(\mathrm{k}_{\mathrm{on}}\right)$ constants.

\subsubsection{Microscale Thermophoresis (MST)}

MST is performed using thin capillaries in free solution, which are illuminated with an infrared laser that generates a temperature gradient. The directed movement of molecules is detected by intrinsic fluorescence, or, in most cases, fluorescent labels of one interactant, and quantified. The thermophoretic movement of molecules within the temperature gradient depends on size, charge, hydration shell or conformation that typically changes upon interaction. The thermophoresis signal is plotted against the ligand concentration to obtain a dose-response curve from which the binding affinity can be deduced [17].

MST screening of the 10 compounds was performed as detailed in the Materials and Methods section (Section 3), and results are reported in Table 1. Analysis revealed that all 
peptides interact with HA with different dissociation constants. In particular, compound 4 binds HA with higher efficiency with respect to 1 (Figure 2), showing an equilibrium dissociation constant $\left(\mathrm{K}_{\mathrm{D}}\right)$ value of $8.22 \pm 0.1 \mathrm{nM}$ and $7.26 \pm 0.06 \mu \mathrm{M}$, respectively. MST binding curves of peptides 2, $\mathbf{3}$ and 5-10 are reported in the Supplementary Materials (Figures S16-S19).

\subsubsection{Surface Plasmon Resonance (SPR)}

For the SPR study, full-length recombinant HA (His-Tag) was immobilized on a sensor chip up to 12,000 response units (RU) (Materials and Methods (Section 3)). Compound binding induced a change in the refractive index on the sensor surface [18]. A regeneration step was necessary (glycine, $\mathrm{pH}$ 1.5, data not shown). After injection, running buffer was allowed to flow over the surface, and the dissociation of compounds from the surface was observed. In contrast, the control flow cell, where no HA was immobilized, showed no significant signal changes (data not shown). The ability of the tetrapeptides to bind HA was defined by their $\mathrm{K}_{\mathrm{D}}$ values (Figures S9-S15, Supplementary Materials).

SPR analysis showed the synthesized peptides efficiently interacting with the immobilized protein. Figure 3 presents the sensorgrams of compounds 1 and 4 bound to HA in HBS-P buffer. Interestingly, tetrapeptide 4 binds HA with higher efficiency with respect to 1 , showing a $K_{D}$ value of $3.57 \pm 0.12 \mathrm{nM}$ and $4.53 \pm 0.08 \mu \mathrm{M}$, respectively.

Both the direct binding measurements demonstrated specific binding between peptide 4 and HA. $K_{\mathrm{D}}$ of the 4: HA complex was $8.22 \pm 0.10 \mathrm{nM}$ in the MST assay and $3.57 \pm 0.12 \mathrm{nM}$ in the SPR assay. This is common as the $\mathrm{K}_{\mathrm{D}}$ values deeply depend on the analysis method and the applied setup.

\subsection{Antiviral Activity}

\subsubsection{Hemagglutination Inhibition Assay (HI)}

The ability of peptides to inhibit HA activity was assessed by HI. The inhibitory effect of peptides $\mathbf{1}$ and $\mathbf{2}$ is reported as a reference. The IAV strains A/Roma-ISS/02/08 H1N1 oseltamivir-sensitive virus, A/Parma/24/09 H1N1 oseltamivir-resistant virus and A/Parma/05/06 H3N2 were used. As shown in Table 1, Ser1, His3 and Ser4 of peptide $\mathbf{1}$ are key amino acids for the antiviral activity against some of the IAV strains used in the assay. The substitution of Ser1 with an alanine determines the loss of activity against the influenza A/Parma/24/09 H1N1 virus subtype of the corresponding analog 3 compared to reference peptide 1 . The absence of positive charge by substitution of Lys2 with Ala determines a significant increase in inhibitory potency of the corresponding analog 4 compared to reference peptide 1 . Peptide 5 increases the inhibitor potency of influenza A/H1N1 strains, showing that His3 is important for inhibitory activity of the influenza A/H3N2 virus subtype. In derivative 6 , the substitution of a hydroxyl chain (Ser4) with a more lipophilic residue (Ala) induces a dramatic loss of activity against the two different influenza A/Parma virus subtypes, increasing the antiviral potency of the influenza A/Roma-ISS/2/08 A/H1N1 viral strain. The data also showed that the substitution of each amino acidic residue of peptide 2 with an alanine determines the loss of activity against the influenza A/Roma-ISS/2/08 H1N1 virus subtype, increasing the antiviral potency on the other two different A/Parma virus subtypes.

Therefore, only one peptide, 4, was able to prevent HA activity of all tested viral strains, in particular, this peptide exerted a strong antiviral action, in the femtomolar range, against two viral strains: A/Roma-ISS/02/08 H1N1 and A/Parma /05/06 H3N2.

\subsubsection{Neutralization Assay (NT)}

Prompted by previous findings, we assessed the ability of peptide 4 to affect virus replication in the Madin-Darby canine kidney (MDCK) cell line by NT. As shown in Table 2, this peptide was able to prevent infection of all tested viruses in a concentration range from about $0.4 \mathrm{fM}$ to $0.9 \mathrm{pM}$, with a relevant antiviral activity against the oseltamivir-resistant $\mathrm{A} / \mathrm{H} 1 \mathrm{~N} 1$ strain with an $\mathrm{EC}_{50}$ value of about $0.4 \mathrm{fM}$ and a very high selectivity index. 
Notably, this peptide was more active against all flu strains compared not only to reference peptide 1 but also to all peptides we studied, starting from the bovine lactoferrin (bLf) C-lobe to all derived peptides $[12,14]$.

Table 2. In vitro antiviral activity of peptides 1 and 4 against influenza virus infection. ${ }^{a} \mathrm{EC}_{50}$ : the reciprocal substance dilution at which $50 \%$ of cells were protected from the virus-induced killing; ${ }^{\wedge} \mathrm{SI}$ : the ratio between $\mathrm{CC}_{50}$ (the reciprocal substance dilution at which $50 \%$ of cells were protected from substance toxicity, corresponding to a concentration $>25 \mu \mathrm{M}$ ) and $\mathrm{EC}_{50}$; The mean values of 3 independent experiments with standard errors are shown. ${ }^{\mathrm{b}}$ Scala et al. (2017) [14].

\begin{tabular}{|c|c|c|c|c|c|c|c|}
\hline \multirow{2}{*}{ Pep. } & \multirow{2}{*}{ Seq. } & \multicolumn{2}{|c|}{ A/Roma-ISS/02/08 H1N1 } & \multicolumn{2}{|c|}{ A/Parma/24/09 H1N1 } & \multicolumn{2}{|c|}{ A/Parma/05/06 H3N2 } \\
\hline & & $\begin{array}{c}E_{50}^{a} \\
(\mu \mathrm{M})\end{array}$ & SI & $\begin{array}{c}\mathrm{EC}_{50}{ }^{\mathrm{a}} \\
(\mu \mathrm{M})\end{array}$ & SI & $\begin{array}{c}E C_{50}{ }^{a} \\
(\mu \mathrm{M})\end{array}$ & SI \\
\hline $1^{b}$ & SKHS & $3 \pm 0.61 \times 10^{-6}$ & $>8.33 \times 10^{6}$ & $4.8 \pm 0.12 \times 10^{-8}$ & $>5.2 \times 10^{8}$ & $5 \pm 0.02 \times 10^{-6}$ & $>5 \times 10^{6}$ \\
\hline $2^{b}$ & SLDC & $5 \pm 0.01 \times 10^{-7}$ & $>5 \times 10^{7}$ & $4.6 \pm 0.05 \times 10^{-6}$ & $>5.4 \times 10^{6}$ & $4.3 \pm 0.03 \times 10^{-6}$ & $>5.8 \times 10^{7}$ \\
\hline 4 & SAHS & $5.77 \pm 0.01 \times 10^{-7}$ & $>4.33 \times 10^{7}$ & $4.3 \pm 0.3 \times 10^{-10}$ & $>5.81 \times 10^{10}$ & $9.36 \pm 0.1 \times 10^{-7}$ & $>2.67 \times 10^{7}$ \\
\hline
\end{tabular}

\subsection{Computational Studies}

To obtain more clues on the tetrapeptide interaction with studied HAs, a structurebased computational analysis was carried out. To be more accurate in the binding prediction, the homology models of our HAs were obtained using the Swiss-Model website (https: / / swissmodel.expasy.org/, accessed on 29 October 2020) on the basis of their nucleotide sequences kindly provided by Dr. Simona Puzelli (Department of Infectious Diseases, ISS, Rome). The obtained model quality was assessed by the QMean and GMQE algorithms (Table S3, Supplementary Materials).

The identification of ligand-binding regions on the HA surface is not trivial because of the large dimension of this trimeric protein and the great variability between and among viral strains. To locate the putative binding sites of our ligands on the HA surface, we evaluated what was suggested from experimental data: HI accounts for the interference with the sialic acid recognition; therefore, the binding in the RBS was evaluated.

The RBS is responsible for the first binding to the sialic acid of glycoproteins on the host cell surface [19]. It represents a relatively conserved region in the receptor-binding domain (RBD) that, on the contrary, is hypervariable to escape host immunity. For this reason, it can provide a useful tool to fight IAV, also because the displacement of the sialic acid is favored by its low $K_{D}$ in the $\mathrm{mM}$ range [20].

A better depiction of the binding site was provided by SiteMap calculation $[21,22]$ that confirmed the hydrophilic character of this site. Different activities between the two A/H1N1 strains can be due to few modified amino acids all around the site: in fact, A/Roma-ISS/02/08 H1N1 has a slightly more flexible loop surrounding the site because of the insertion of an additional Ala125 (alignment of A/Roma and A/Parma H1N1 sequences is reported in the Supplementary Materials (Figure S21).

The docking calculations were carried out setting the SP-peptide docking procedure available in Glide [22-25] aimed to increase the conformational exploration of ligands during the docking process, retrieving up to 100 docked poses. This procedure is particularly useful to have a picture of possible binding modes of very flexible molecules such as our peptides. Because of the large number of resulting geometries, these were clustered to select the most reliable binding pose for each ligand, taking into account both docking score and number of retrieved similar conformations, so the highest scoring ligand geometry of the most populated cluster is reported in the figures.

For the sake of simplicity, only the docked poses of the most active compound 4 and reference peptide $\mathbf{1}$ in the RBS of the three HAs are reported (Figures 4 and 5). A similar binding mode was obtained for the other ligands. Binding geometries of peptide 2 are represented in Figure S22 of the Supplementary Materials as a broad-spectrum representative of the second series of tetrapeptides. The total view of the HA-peptide complex is shown for compound 4 in the Parma/H1N1 (Figure 4A) as a representative. 
The ligand 4 has a conserved binding geometry in the three studied HAs, spanning the whole site. RBS residues involved in H-bond interactions with our ligands are the same that get into contact with the sialic acid in most cases; in particular, residues corresponding to Asp174, Glu211, Gln210, Tyr80 and Thr118 (A/Parma H1N1 numbering) are responsible for binding with 6'-SLN (PDB ID 3UBN [26]). Most involved ligand residues are the two serine residues forming a network of $\mathrm{H}$-bonds. One of them, in particular, displaces a conserved water molecule with its OH group (Figure S23, Supplementary Materials).

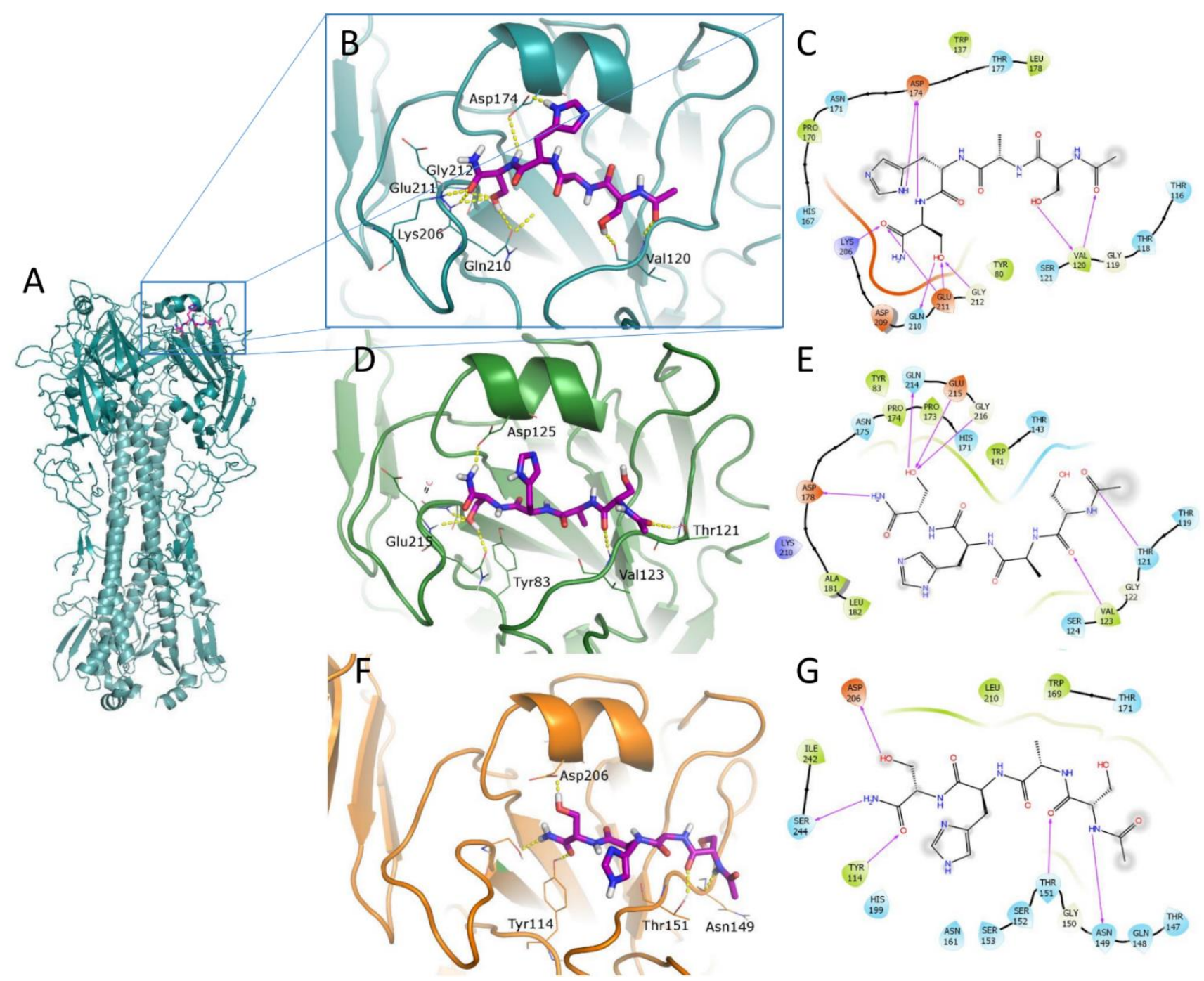

Figure 4. Docked poses of peptide 4 (purple C atoms, represented as sticks) in the RBS of studied HAs represented as cartoons. (A) Total view of Parma/H1N1 (deep-cyan HA1 chains, pale cyan HA2 chains); zoomed view of: (B) Parma/H1N1 (deep cyan); (D) Roma/H1N1 (dark green); (F) Parma/H3N2 (orange). Residues involved in H-bond interactions with the ligand are represented as lines; H-bonds are depicted as yellow dashed lines. Corresponding 2D ligand interaction diagrams are reported in panels $(\mathbf{C}, \mathbf{E}, \mathbf{G})$; in these latter diagrams, residues close to the ligand are colored on the basis of their properties (orange, negatively charged; blue, positively charged; green, hydrophobic; cyan, polar), H-bonds are depicted as magenta arrows, solvent-exposed atoms are surrounded by a gray shadow. 


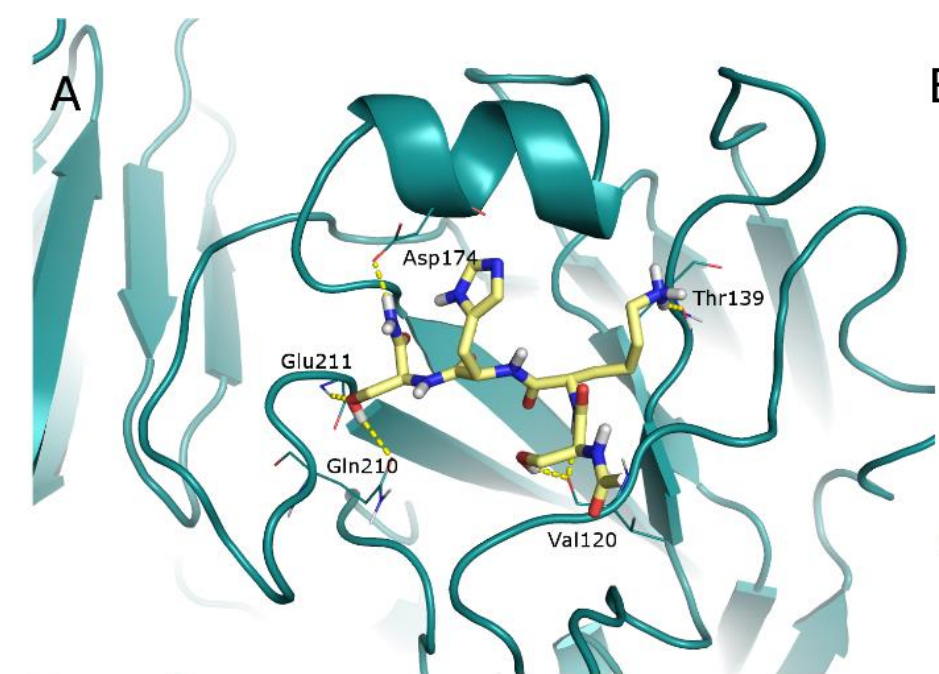

B
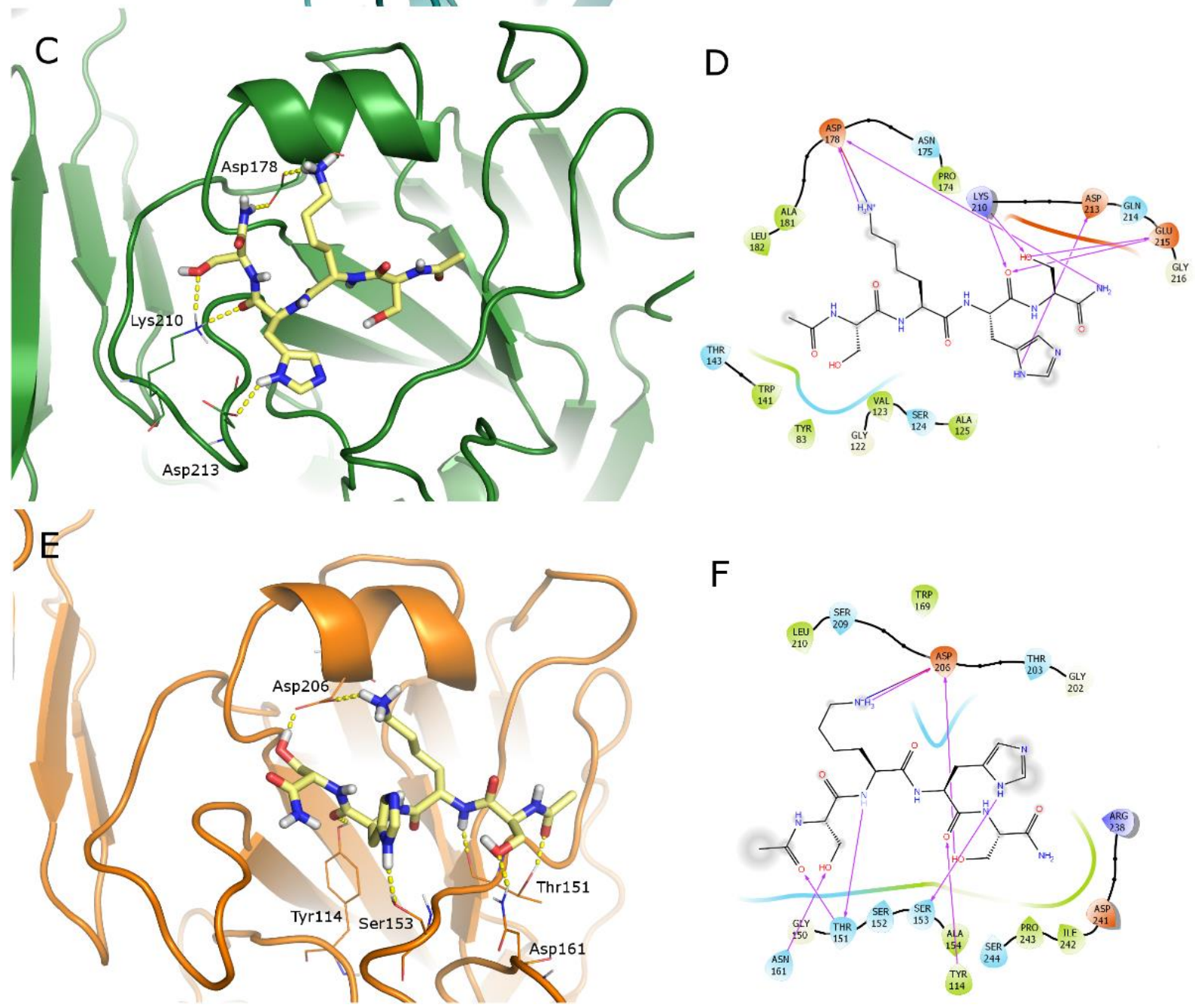

$\mathrm{F}$
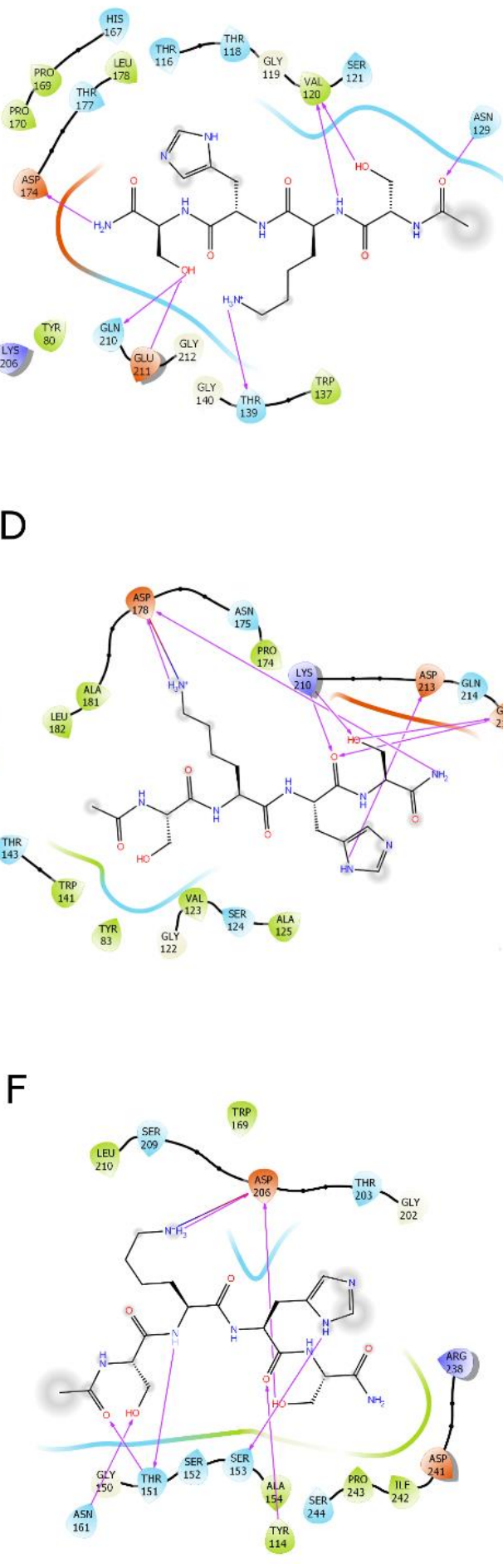

Figure 5. Docked poses of peptide 1 (yellow C atoms, represented as sticks) in the RBS of studied HAs represented as cartoons: (A) Parma/H1N1 (deep-cyan); (C) Roma/H1N1 (dark green); (E) Parma/H3N2 (orange). Residues involved in $\mathrm{H}$-bond interactions with the ligand are represented as lines; H-bonds are depicted as yellow dashed lines. Corresponding 2D ligand interaction diagrams are reported in panels $(\mathbf{B}, \mathbf{D}, \mathbf{F})$; in the diagrams, residues close to the ligand are colored on the basis of their properties (orange, negatively charged; blue, positively charged; green, hydrophobic; cyan, polar), H-bonds are depicted as magenta arrows, solvent-exposed atoms are surrounded by a gray shadow. 
For an explanation of the higher activity observed for peptide 4 compared to its precursor 1, the docked poses of this latter are reported in Figure 5. The analysis of the retrieved geometries in the RBS does not provide a clear justification: peptide $\mathbf{1}$ shows a similar occupation of the binding sites with the ligand in an extended conformation and forms a pattern of interactions with the residues engaged with 4 . Nevertheless, the long and flexible side chain of Lys residue modifies the binding geometries with respect to peptide 4 .

Some clues can be obtained by evaluating the ligand strain energy, i.e., the energy the ligands spent to reach the binding geometries from its minimum. Data reported in Table 3 suggest that peptide 1 makes a larger effort to reach the docking geometries compared to ligand 4, probably because of the flexibility of the Lys side chain, which is also reflected in the docking score value. This aspect can contribute to explaining the improved activity of compound 4 with respect to 1 .

Table 3. Calculated strain energy and docking score of docked poses of peptides $\mathbf{1}$ and $\mathbf{4}$ in the studied HAs. These values are obtained running both a constrained and an unconstrained minimization with MacroModel. The energy difference is used to determine the strain energy.

\begin{tabular}{cccc}
\hline HA Subtype & Pep. & Strain Energy & Docking Score \\
\hline \multirow{2}{*}{ Roma/H1N1 } & $\mathbf{1}$ & 6.682 & -5.174 \\
& $\mathbf{4}$ & 2.525 & -7.554 \\
Parma/H1N1 & $\mathbf{1}$ & 9.014 & -5.266 \\
& $\mathbf{4}$ & 4.050 & -6.259 \\
Parma/H3N2 & $\mathbf{1}$ & 6.958 & -5.230 \\
& $\mathbf{4}$ & 2.081 & -6.137 \\
\hline
\end{tabular}

\section{Materials and Methods}

\subsection{Synthesis}

$\mathrm{N} \alpha$-Fmoc-protected amino acids, Rink amide resin, coupling reagents, $\mathrm{N}, \mathrm{N}$-diisopropylethylamine (DIEA), piperidine and trifluoroacetic acid (TFA) were purchased from Iris Biotech (Marktredwitz, Germany). Peptide synthesis solvents, reagents and $\mathrm{CH}_{3} \mathrm{CN}$ for high-performance liquid chromatography (HPLC) were reagent grade, acquired from commercial sources and used without further purification unless otherwise noted.

\subsubsection{Peptide Synthesis}

The synthesis of tetrapeptides (1-10) was performed according to the solid-phase approach using standard 9-fluorenylmethoxycarbonyl (Fmoc) methodology $[27,28]$ in a manual reaction vessel on Rink amide resin $(0.150 \mathrm{~g}, 0.7 \mathrm{mmol} / \mathrm{g}$ loading $)$ previously Fmoc-deprotected by $25 \%$ piperidine solution in DMF $(1 \times 5 \mathrm{~min}$ and $1 \times 25 \mathrm{~min})$. Each coupling reaction was accomplished using a 3-fold excess of amino acid with 2(1H-benzotriazole-1-yl)-1,1,3,3-tetramethyluronium hexafluorophosphate (HBTU) and 1hydroxybenzotriazole (HOBt) in the presence of DIEA (6 eq.). The peptide resin was washed with dichloromethane (DCM, $3 \times$ ), N,N-dimethylformamide (DMF, $3 \times$ ) and DCM $(3 \times)$, and the Fmoc deprotection protocol, described above, was repeated after each coupling step. After peptide assembly, the N-terminal Fmoc group was removed, and the peptides were acetylated by adding a solution of $\mathrm{Ac}_{2} \mathrm{O} / \mathrm{DCM}$ (1:3) and shaking for $30 \mathrm{~min}$. Finally, peptides were released from the resin using a cleavage mixture containing $90 \%$ TFA, $5 \%$ triisopropylsilane (TIS) and $5 \% \mathrm{H}_{2} \mathrm{O}$ for $2 \mathrm{~h}$. The resin was removed by filtration, and the crude peptide was recovered by precipitation with cold anhydrous ethyl ether to give a white powder and then lyophilized.

\subsubsection{Purification and Characterization}

All crude peptides were purified by RP-HPLC on a preparative C18-bonded silica column (Phenomenex Kinetex AXIA $100 \AA, 100 \times 21.2 \mathrm{~mm}, 5 \mu \mathrm{m}$ ) using a Shimadzu SPD 20 A UV/VIS detector, with detection at 214 and $254 \mathrm{~nm}$. Mobile phase was: (A) $\mathrm{H}_{2} \mathrm{O}$ and 
(B) ACN, both acidified with $0.1 \%$ TFA $(v / v)$. Injection volume was $5000 \mu \mathrm{L}$; flow rate was set to $15 \mathrm{~mL} / \mathrm{min}$. The following gradient was employed: 0-18 $\mathrm{min}, 1-40 \% \mathrm{~B}, 18.01-20 \mathrm{~min}$, $40-70 \%$ B, 20.01-21 min, 70-90\% B, 21.01-23 min, returning to 1\% B. Analytical purity and retention time (tr) of each peptide were determined using HPLC conditions in the above solvent system (solvents A and B) programmed at a flow rate of $0.800 \mathrm{~mL} / \mathrm{min}$, fitted with a Supelco C-18 column and an Ascentis express peptide C18 column $(50 \times 3.00 \mathrm{~mm}, 2.7 \mu \mathrm{m})$. LC gradient was the following: 0-7 $\mathrm{min}, 1-40 \% \mathrm{~B}, 7.01-8 \mathrm{~min}, 40-90 \% \mathrm{~B}, 8.01-9 \mathrm{~min}$, returning to $1 \% \mathrm{~B}, 9-11 \mathrm{~min}$, isocratic for $2 \mathrm{~min}$. All analogs showed $>97 \%$ purity when monitored at $220 \mathrm{~nm}$. Homogeneous fractions, as established using analytical HPLC, were pooled and lyophilized.

Ultra-high-resolution mass spectra were obtained by positive ESI infusion on an LTQ Orbitrap XL mass spectrometer (Thermo Scientific, Dreieich, Germany), equipped with Xcalibur software for processing the data acquired. The sample was dissolved in a mixture of water and methanol (50/50) and injected directly into the electrospray source, using a syringe pump, at constant flow $(15 \mu \mathrm{L} / \mathrm{min})$. The temperature of the capillary was set at $220{ }^{\circ} \mathrm{C}$.

\subsection{Direct Binding Assay}

HA was purchased from GenScript, Cat No.: Z03181-100. CM5 sensor chips, HBSP+ buffer (0.01 M HEPES pH 7.4, 0.15 M NaCl, 0.05\% v/v Surfactant P20), 1-ethyl-3(3-diaminopropyl) carbodiimide hydrochloride (EDC), N-hydroxysuccinimide (NHS), ethanolamine $\left(\mathrm{H}_{2} \mathrm{~N}\left(\mathrm{CH}_{2}\right)_{2} \mathrm{OH}\right)$ and regeneration solution were purchased from Cytiva.

\subsubsection{Microscale Thermophoresis (MST)}

MST experiments were carried out using the Monolith NT.115pico instrument (NanoTemper Technologies, Munich, Germany). His-HA was labeled using the Nanotemper His-Tag Labeling Kit RED-tris-NTA 2nd Generation as described elsewhere [29]. Briefly, $100 \mu \mathrm{L}$ of a solution of His-HA protein $(80 \mathrm{nM})$ in double-distilled water was mixed with $100 \mu \mathrm{L}$ of 40 nM NT647-NHS fluorophore (NanoTemper Technologies, Munich, Germany) in labeling buffer and incubated for $30 \mathrm{~min}$ at room temperature. NT647-HA was centrifuged for $10 \mathrm{~min}$ at $4{ }^{\circ} \mathrm{C}$ at $15,000 \times g$ to remove protein aggregates. Pretests using standard-treated and premium-coated MST capillaries (NanoTemper Technologies, Munich, Germany) were performed to test the adsorption of labeled HA to capillary walls by analyzing capillary scans recorded by the Monolith NT.115pico prior to MST experiments [30]. The protein did not adsorb to standard-treated capillary walls in assay buffer (PBS). For this reason, the following experiments were performed using a standard-treated capillary. Then, buffer conditions were evaluated to identify the optimal state for MST signal reproducibility and the suppression of unspecific adsorption to capillary walls. Compound stocks $(5 \mathrm{mM})$ in MST buffer were diluted in the assay buffer to reach the highest soluble concentration (50 $\mathrm{MM}$ ). In MST experiments, 16-fold 1:1 serial dilutions of each compound were mixed with NT647-HA to yield a final reaction volume of $20 \mu \mathrm{L}$. After $10 \mathrm{~min}$ of incubation at $\mathrm{rt}$, the reaction mixtures were loaded into standard-treated capillaries and subsequently inserted in the chip tray of Monolith NT.115 for thermophoresis analysis and the appraisal of $K_{D}$ values. Signals were recorded at high MST power and 10\% LED power. $\mathrm{K}_{\mathrm{D}}$ values were calculated from compound concentration-dependent changes in normalized fluorescence $\left(\mathrm{F}_{\text {norm}}\right)$ of $\mathrm{HA}$ after $21 \mathrm{~s}$ of thermophoresis. Each compound was tested in triplicate and data analyzed using MO Affinity Analysis software (NanoTemper Technologies, Munich, Germany). Confidence values $( \pm)$ were indicated next to the $K_{D}$ value for each tested compound.

\subsubsection{Surface Plasmon Resonance (SPR)}

SPR binding studies were performed at $25^{\circ} \mathrm{C}$ using Biacore T200 (Cytiva, Uppsala, SwedenHis-HA were stably captured at the surface of the CM5 sensor chip by means of an antihistidine antibody (His Capture Kit, Cytiva) that had been covalently bound to the 
surface as recommended by the manufacturer. In particular, the antihistidine antibody provided in the His Capture Kit was diluted to $50 \mu \mathrm{g} / \mathrm{mL}$ in the immobilization buffer included in the kit and covalently coupled to Sensor Chip CM5 by standard amine coupling to a level of approximately $12,000 \mathrm{RU}$. Then, His-HA was injected $\left(21,5 \mu \mathrm{g} \cdot \mathrm{mL}^{-1} 10 \mathrm{~mm}\right.$ acetate, $\mathrm{pH} 4.59$ ) over the antihistidine antibody surface for $1 \mathrm{~min}$. No protein was injected over the reference surface. The dissociation was monitored by injecting running buffer for $600 \mathrm{~s}$. Surface regeneration was performed by injecting glycine buffer $(10 \mathrm{mM}, \mathrm{pH} 1.5$, $1 \mathrm{~min})$.

HBS-P+ buffer was used as running buffer. After the immobilization of HA, HBS-P+ buffer was injected over the chip at a flow rate of $5 \mu \mathrm{L} / \mathrm{min}$ overnight. A solution of peptide in HBS-P+ buffer at various concentrations (from 0.32 to $10 \mu \mathrm{M}$ ) was injected at $25^{\circ} \mathrm{C}$ with a flow rate of $30 \mu \mathrm{L} / \mathrm{min}$ for $120 \mathrm{~s}$ (association phase), and then the buffer alone was injected for $600 \mathrm{~s}$ (dissociation phase).

The equilibrium dissociation constants $\left(\mathrm{K}_{\mathrm{D}}\right)$ and kinetic dissociation $(\mathrm{kd})$ and association $(\mathrm{ka})$ constants were calculated from the sensorgrams by global fitting of a 1:1 binding model using BIAevaluation software (v3.1) provided with the Biacore T200 instrument (Cytiva).

\subsection{Biological Assay}

\subsubsection{Cells and Viral Strains}

Madin-Darby canine kidney (MDCK, ATCC, CRL-2936) cells were grown at $37^{\circ} \mathrm{C}$ in minimal essential medium (MEM, Invitrogen, Paisley, UK) containing $1.2 \mathrm{~g} / \mathrm{L} \mathrm{NaHCO}_{3}$ and supplemented with 10\% inactivated fetal calf serum (FCS, Invitrogen, Paisley, UK), $2 \mathrm{mM}$ glutamine, nonessential amino acids, penicillin $(100 \mathrm{IU} / \mathrm{mL})$ and streptomycin $(100 \mu \mathrm{g} / \mathrm{mL})$. The following IAV strains were used: A/RomaISS/02/08 H1N1 (Brisbane-like) oseltamivirsensitive virus, A/Parma/24/09 H1N1 (Brisbane-like) oseltamivir-resistant virus and A/Parma/05/06 H3N2 (Wisconsin-like) virus. Viruses were propagated in MDCK cells in serum-free MEM supplemented with $4 \%$ bovine serum albumin (BSA fraction V, Gibco; Paisley, UK), $1 \mu \mathrm{g} / \mu \mathrm{L}$ N-tosyl-L-phenylalanine chloromethyl ketone-treated trypsin (Sigma Chemical Co.; St. Louis, MO, USA). When an extensive cytopathic effect (c.p.e.) was observed, infected cultures were frozen and thawed three times, centrifuged (3000 rpm, $10 \mathrm{~min}$ ), and supernatants were stored at $-80^{\circ} \mathrm{C}$. Titers of virus stocks were determined by hemagglutinin titration and/or plaque assay according to the standard procedures [31,32].

\subsubsection{Cytotoxicity Assay}

This procedure was performed as reported elsewhere [33]. Briefly, two-fold serial dilutions of each peptide in culture medium were incubated at $37^{\circ} \mathrm{C}$ with confluent MDCK cells grown in 96-well tissue culture microplates (Nalge Nunc Europe Ltd., Neerijse, Belgium). After $24 \mathrm{~h}$, the following parameters were evaluated: cell morphology, which was examined by light microscopy; cell viability, which was determined by neutral red staining as already described by us [34]; and cell proliferation, which was evaluated quantitatively by microscopic counts after dispersion into individual cells with trypsin. Peptide dilutions that did not affect any of these parameters were considered noncytotoxic concentrations and utilized for antiviral assays.

\subsubsection{Hemagglutination Inhibition Assay (HI)}

Viruses in phosphate-buffered saline (PBS, pH 7.4) were incubated for $1 \mathrm{~h}$ at $4{ }^{\circ} \mathrm{C}$ with serial dilutions of peptides in PBS. An equal volume of $0.5 \%$ turkey erythrocytes was then added and allowed to agglutinate. Titers were expressed as the reciprocal of the peptide dilutions giving $50 \%$ hemagglutination of erythrocytes by four virus-agglutinating units.

\subsubsection{Neutralization Assay (NT)}

NT was carried out by incubating serial two-fold peptide dilutions, starting from $12.5 \mu \mathrm{M}$, in culture medium with equal volumes of viral suspension containing $10^{6}$ plaque- 
forming units (p.f.u.) for $1 \mathrm{~h}$ at $4{ }^{\circ} \mathrm{C}$. In negative controls, culture medium was used instead of peptides in the same volume. MDCK cells, grown in 96-well tissue culture microplates (Nalge Nunc Europe Ltd., Neerijse, Belgium), were infected with $100 \mu \mathrm{L} /$ well (10 p.f.u./cell; in quadruplicate) of the virus-peptide mixtures. After adsorption, cells were rinsed thoroughly and incubated at $37^{\circ} \mathrm{C}$ for $24 \mathrm{~h}$. The viral c.p.e. was measured by neutral red staining as reported elsewhere by our laboratory [33].

\subsection{Computational Studies}

\subsubsection{Homology Modeling}

Homology models for the three viral strains were generated using the Swiss Model webserver (https: / / swissmodel.expasy.org/, accessed on 29 October 2020) [35]. The FASTA nucleotide sequence of the three HAs (A/Parma H1N1, A/Roma H1N1, A/Parma H3N2) was used to search for the best-matching proteins. To obtain the trimeric form, the quaternary structure annotation was introduced in the FASTA sequence. Suitable templates were aligned using the Basic Local Alignment Search Tool (BLAST) [36] and Hidden Markov model-based lightning-fast iterative sequence search (HHblits) [37]. The highest-ranking template was selected to build the protein models using ProMod3 [38]. The quality of obtained models was assessed using Qualitative Model Energy ANalysis (QMEAN) [39] and Global Model Quality Estimate (GMQE). The Ramachandran plot for each obtained structure was generated to determine the stereochemical and conformational quality (Figure S20, Supplementary Materials). The validity of the obtained quaternary structure was provided by the Quaternary Structure Quality Estimate (QSQE) score [40]. Identified templates and related score values for the three models are reported in the Supplementary Materials (Tables S2 and S3).

\subsubsection{Protein Preparation}

The three proteins were aligned to each other in Maestro using the Protein Structure Alignment tool that performs the process of aligning the protein sequences.

Superimposed homology models were submitted to the Protein Preparation routine in Maestro in order to optimize the obtained structures. In particular, the H-bond optimizer and the restrained minimization were carried out to ameliorate the H-bond network.

\subsubsection{Binding Site Identification and Analysis}

To locate the putative binding site on the receptor-binding domain of the studied HAs, the homology models were aligned to the available X-ray complex of pandemic HA bound to $6^{\prime}$-SLN (PDB ID: 3UBN, res $2.51 \AA$ [26]).

Each putative site was explored by performing a SiteMap [21,22] calculation using the following settings: evaluate a single binding site using the aligned X-ray ligand as a reference. The fine grid and more restrictive definition of hydrophobicity were applied. The site was cropped at $6 \AA$ from the last site point.

\subsubsection{Receptor Grid Generation}

For all sites mentioned in the previous paragraph, the receptor grid was generated using the Receptor Grid Generation routine available in Glide [22-25]. The grid box was enlarged to dock ligands $\leq 20 \AA$, and the inner box size was set to: $x=10, y=15, z=10$. All grids were generated with settings suitable to peptide docking.

All sites in the RBD were located exploiting the SiteMap calculated site points The following rotatable groups were set: Thr147, Thr151, Ser152, Ser153, Thr171, Thr203, Ser209 for Parma/H3N2; Tyr80, Ser122, Thr177, Tyr179 for A/Parma H1N1 and Tyr83, Thr121, Se133, Thr143, Tyr183 for Roma/H1N1.

\subsubsection{Ligand Preparation}

The structures of the studied peptides 1-10 were built in Maestro using the Build tool. Obtained structures were submitted to Ligprep to generate possible tautomers and 
protomers at physiological $\mathrm{pH}(7.0 \pm 0.4)$. Resulting structures were minimized to a derivative convergence of $0.001 \mathrm{~kJ} / \mathrm{mol}^{-1}$ using the PRCG minimization algorithm, the OPLS3e force field and the generalized Born/surface area (GB/SA) water solvation model implemented in MacroModel [22].

\subsubsection{Docking Calculations}

Minimized ligand structures were docked in all the previously identified sites on the HA RBD using Glide and the SP-peptide docking protocol that allows increasing the conformational exploration of ligands, affording a final set of 100 poses. Obtained poses for each ligand were clustered using the Clustering of Conformers tool available in Maestro. The Average clustering algorithm was used on the basis of the RMSD calculated between heavy atom pairs. The Kelley penalty was calculated, and the optimal clustering level was set consequently. Most populated clusters were evaluated along with the docking score. To calculate the docked poses strain energy, the corresponding tool available in Maestro was used that performed a constrained and full minimization of the docked poses of ligands 1 and 4 in the three studied HAs. The default parameters were applied in the calculation.

\section{Conclusions}

In this paper, we performed a systematic SAR study through the development of ala-scan peptides starting from the most promising tetrapeptides identified previously [14]. This study allowed us to assess the importance of the side chain on peptide bioactivity.

Direct binding assays were carried out exploiting two biophysical methods (MST and SPR), while the evaluation of antiviral activity was assessed through both HI and NT studies. Structure-based computational studies allowed us to envision the putative interactions of this ligand with HA, highlighting the role of serine residues in receptor binding. All applied methods agreed upon the identification of a novel potent tetrapeptide, Ac-SAHS- $\mathrm{NH}_{2}$, able to bind hemagglutinin with high affinity and inhibit influenza virus hemagglutination and cell infection at femtomolar concentration. This small sequence, with high and broad-spectrum activity, can represent a valuable starting point for the design of small molecules. The work carried out opens the way to new perspectives for the development of new anti-influenza drugs, especially in a context in which the emergence of new and drug-resistant viruses highlights the need for new antiviral approaches and strategies.

Supplementary Materials: The following are available online at https:/ / www.mdpi.com/article/10 .3390/ph14100959/s1, Table S1. Analytical data of peptides 1-10; Figures S1-S8. HRMS spectra and HPLC chromatograms of peptide 3-10; Figures S9-S15. Sensor grams of peptides 3, 5-10; Figures S16-S19. MST binding curves of peptides 2, 3, 5-10; Table S2. Swiss-Model data of homology models; Table S3. Score values obtained for the best homology models; Figure S20. Ramachandran Plots of HA homology models; Figure S21. Alignment of HA sequences of A/Parma and A/Roma H1N1 strains; Figure S22. Docked poses of peptide 2; Figure S23. Superposition of the complex of HA: sialic acid with docked pose of SAHS.

Author Contributions: M.S. and P.C.: supervision; M.S.: conceptualization and project administration. M.S. and M.C.S.: biophysical assay; M.S., M.C.S., M.A. and F.S.: data curation, writing-original draft preparation, formal analysis; M.C.S. and V.D.S.: methodology, synthesis; M.A.: computational studies; M.S., M.A., F.S. and P.C.: writing-review; A.P. and F.S.: biological assay; A.B.: resources; P.C.: funding acquisition and editing. All authors have read and agreed to the published version of the manuscript.

Funding: This work was supported by Grants from the National Institute of Health (Italy) "Studi sull'attività antimicrobica di sostanze di origine naturale o sintetiche" (Grant numbers: R942) (F.S.).

Institutional Review Board Statement: Not applicable.

Informed Consent Statement: Not applicable.

Data Availability Statement: Data are within the article and Supplementary Materials. 
Acknowledgments: The assistance of the staff is gratefully appreciated.

Conflicts of Interest: The authors declare no conflict of interest.

\section{References}

1. Hale, B.G.; Albrecht, R.A.; García-Sastre, A. Innate immune evasion strategies of influenza viruses. Future Microbiol. 2010, 5, 23-41. [CrossRef]

2. Shi, W.; Gao, G.F. Emerging H5N8 avian influenza viruses. Science 2021, 372, 784-786. [CrossRef]

3. Hayden, F.G. Respiratory viral threats. Curr. Opin. Infect. Dis. 2006, 19, 169-178. [CrossRef]

4. de St Maurice, A.; Halasa, N. Preparing for the 2019-2020 influenza season. Pediatr. Transplant. 2020, 24, e13645. [CrossRef] [PubMed]

5. Alves Galvão, M.G.; Rocha Crispino Santos, M.A.; Alves da Cunha, A.J. Amantadine and rimantadine for influenza A in children and the elderly. Cochrane Database Syst. Rev. 2014, 11, CD002745. [CrossRef]

6. Kamali, A.; Holodniy, M. Influenza treatment and prophylaxis with neuraminidase inhibitors: A review. Infect. Drug. Resist. 2013, 6, 187-198. [CrossRef] [PubMed]

7. Shie, J.J.; Fang, J.M. Development of effective anti-influenza drugs: Congeners and conjugates-A review. J Biomed Sci. 2019, 26, 84. [CrossRef]

8. Aschenbrenner, D.S. Xofluza Now Indicated to Prevent Influenza. Am. J. Nurs. 2021, 121, 26-27. [CrossRef] [PubMed]

9. Belshe, R.B.; Burk, B.; Newman, F.; Cerruti, R.L.; Sim, I.S. Resistance of influenza A virus to amantadine and rimantadine: Results of one decade of surveillance. J. Infect. Dis. 1989, 159, 430-435. [CrossRef] [PubMed]

10. Bertram, S.; Glowacka, I.; Steffen, I.; Kühl, A.; Pöhlmann, S. Novel insights into proteolytic cleavage of influenza virus hemagglutinin. Rev. Med. Virol. 2010, 20, 298-310. [CrossRef]

11. Bullard, B.L.; Weaver, E.A. Strategies Targeting Hemagglutinin as a Universal Influenza Vaccine. Vaccines 2021, 9, 257. [CrossRef]

12. Ammendolia, M.G.; Agamennone, M.; Pietrantoni, A.; Lannutti, F.; Siciliano, R.A.; De Giulio, B.; Amici, C.; Superti, F. Bovine lactoferrin-derived peptides as novel broad-spectrum inhibitors of influenza virus. Pathog. Glob. Health 2012, 106, 12-19. [CrossRef]

13. Superti, F.; Agamennone, M.; Pietrantoni, A.; Ammendolia, M.G. Bovine Lactoferrin Prevents Influenza A Virus Infection by Interfering with the Fusogenic Function of Viral Hemagglutinin. Viruses 2019, 11, 51. [CrossRef]

14. Scala, M.C.; Sala, M.; Pietrantoni, A.; Spensiero, A.; Di Micco, S.; Agamennone, M.; Bertamino, A.; Novellino, E.; Bifulco, G.; Gomez-Monterrey, I.M.; et al. Lactoferrin-derived Peptides Active towards Influenza: Identification of Three Potent Tetrapeptide Inhibitors. Sci. Rep. 2017, 7, 10593. [CrossRef] [PubMed]

15. Gomez-Monterrey, I.; Sala, M.; Rusciano, M.R.; Monaco, S.; Maione, A.S.; Iaccarino, G.; Tortorella, P.; D’Ursi, A.M.; Scrima, M.; Carotenuto, A.; et al. Characterization of a selective CaMKII peptide inhibitor. Eur. J. Med. Chem. 2013, 62, 425-434. [CrossRef] [PubMed]

16. Renaud, J.P.; Chung, C.W.; Danielson, U.H.; Egner, U.; Hennig, M.; Hubbard, R.E.; Nar, H. Biophysics in drug discovery: Impact, challenges and opportunities. Nat. Rev. Drug. Discov. 2016, 15, 679-698. [CrossRef] [PubMed]

17. Duhr, S.; Braun, D. Why molecules move along a temperature gradient. Proc. Natl. Acad. Sci. USA 2006, 103, 19678-19682. [CrossRef]

18. Navratilova, I.; Hopkins, A.L. Fragment screening by surface plasmon resonance. ACS Med. Chem. Lett. 2010, 1, 44-48. [CrossRef]

19. Weis, W.; Brown, J.H.; Cusack, S.; Paulson, J.C.; Skehel, J.J.; Wiley, D.C. Structure of the influenza virus haemagglutinin complexed with its receptor, sialic acid. Nature 1988, 333, 426-431. [CrossRef]

20. Kadam, R.U.; Wilson, I.A. A small-molecule fragment that emulates binding of receptor and broadly neutralizing antibodies to influenza A hemagglutinin. Proc. Natl. Acad. Sci. USA 2018, 115, 4240-4245. [CrossRef]

21. Halgren, T.A. Identifying and characterizing binding sites and assessing druggability. J. Chem. Inf. Model. 2009, 49, 377-389. [CrossRef] [PubMed]

22. Schrödinger LLC. Schrödinger Release, 2020-2024: Maestro, Glide, LigPrep, MacroModel, SiteMap; Schrödinger, LLC: New York, NY, USA, 2020.

23. Friesner, R.A.; Banks, J.L.; Murphy, R.B.; Halgren, T.A.; Klicic, J.J.; Mainz, D.T.; Repasky, M.P.; Knoll, E.H.; Shelley, M.; Perry, J.K.; et al. Glide: A new approach for rapid, accurate docking and scoring. 1. Method and assessment of docking accuracy. J. Med. Chem. 2004, 47, 1739-1749. [CrossRef] [PubMed]

24. Halgren, T.A.; Murphy, R.B.; Friesner, R.A.; Beard, H.S.; Frye, L.L.; Pollard, W.T.; Banks, J.L. Glide: A new approach for rapid, accurate docking and scoring. 2. Enrichment factors in database screening. J. Med. Chem. 2004, 47, 1750-1759. [CrossRef] [PubMed]

25. Friesner, R.A.; Murphy, R.B.; Repasky, M.P.; Frye, L.L.; Greenwood, J.R.; Halgren, T.A.; Sanschagrin, P.C.; Mainz, D.T. Extra precision glide: Docking and scoring incorporating a model of hydrophobic enclosure for protein-ligand complexes. J. Med. Chem. 2006, 49, 6177-6196. [CrossRef]

26. Xu, R.; McBride, R.; Nycholat, C.M.; Paulson, J.C.; Wilson, I.A. Structural characterization of the hemagglutinin receptor specificity from the 2009 H1N1 influenza pandemic. J. Virol. 2012, 86, 982-990. [CrossRef]

27. Atherton, E.; Sheppard, R.C. Solid-Phase Peptide Synthesis: A Practical Approach; Oxford University Press: Oxford, UK, 1989. 
28. Pescina, S.; Sala, M.; Padula, C.; Scala, M.C.; Spensiero, A.; Belletti, S.; Gatti, R.; Novellino, E.; Campiglia, P.; Santi, P.; et al. Design and Synthesis of New Cell Penetrating Peptides: Diffusion and Distribution Inside the Cornea. Mol. Pharm. 2016, 13, $3876-3883$. [CrossRef]

29. Monolith NT Protein Labeling Kit RED-NHS. User Manual. Available online: https:/ / manualzz.com/doc/7334293/monolithnt $\%$ E2\%84\%A2-protein-labeling-kit-red-nhs (accessed on 29 October 2020).

30. Milite, C.; Feoli, A.; Horton, J.R.; Rescigno, D.; Cipriano, A.; Pisapia, V.; Viviano, M.; Pepe, G.; Amendola, G.; Novellino, E.; et al. Discovery of a Novel Chemotype of Histone Lysine Methyltransferase EHMT1/2 (GLP/G9a) Inhibitors: Rational Design, Synthesis, Biological Evaluation, and Co-crystal Structure. J. Med. Chem. 2019, 62, 2666-2689. [CrossRef]

31. Gaush, C.R.; Smith, T.F. Replication and plaque assay of influenza virus in an established line of canine kidney cells. Appl. Microbiol. 1968, 16, 588-594. [CrossRef]

32. Rimmelzwaan, G.F.; Baars, M.; Claas, E.C.; Osterhaus, A.D. Comparison of RNA hybridization, hemagglutination assay, titration of infectious virus and immunofluorescence as methods for monitoring influenza virus replication in vitro. J. Virol. Methods 1998, 74, 57-66. [CrossRef]

33. Pietrantoni, A.; Dofrelli, E.; Tinari, A.; Ammendolia, M.G.; Puzelli, S.; Fabiani, C.; Donatelli, I.; Superti, F. Bovine lactoferrin inhibits influenza A virus induced programmed cell death in vitro. Biometals 2010, 23, 465-475. [CrossRef]

34. Marchetti, M.; Trybala, E.; Superti, F.; Johansson, M.; Bergström, T. Inhibition of herpes simplex virus infection by lactoferrin is dependent on interference with the virus binding to glycosaminoglycans. Virology 2004, 318, 405-413. [CrossRef]

35. Waterhouse, A.; Bertoni, M.; Bienert, S.; Studer, G.; Tauriello, G.; Gumienny, R.; Heer, F.T.; de Beer, T.; Rempfer, C.; Bordoli, L.; et al. SWISS-MODEL: Homology modelling of protein structures and complexes. Nucleic Acids Res. 2018, 46, W296-W303. [CrossRef]

36. Camacho, C.; Coulouris, G.; Avagyan, V.; Ma, N.; Papadopoulos, J.; Bealer, K.; Madden, T.L. BLAST+: Architecture and applications. BMC Bioinform. 2009, 10, 421. [CrossRef]

37. Remmert, M.; Biegert, A.; Hauser, A.; Söding, J. HHblits: Lightning-fast iterative protein sequence searching by HMM-HMM alignment. Nat. Methods 2012, 9, 173-175. [CrossRef] [PubMed]

38. Biasini, M.; Bienert, S.; Waterhouse, A.; Arnold, K.; Studer, G.; Schmidt, T.; Kiefer, F.; Gallo Cassarino, T.; Bertoni, M.; Bordoli, L.; et al. SWISS-MODEL: Modelling protein tertiary and quaternary structure using evolutionary information. Nucleic Acids Res. 2014, 42, W252-W258. [CrossRef] [PubMed]

39. Benkert, P.; Biasini, M.; Schwede, T. Toward the estimation of the absolute quality of individual protein structure models. Bioinformatics 2011, 27, 343-350. [CrossRef] [PubMed]

40. Bertoni, M.; Kiefer, F.; Biasini, M.; Bordoli, L.; Schwede, T. Modeling protein quaternary structure of homo- and hetero-oligomers beyond binary interactions by homology. Sci. Rep. 2017, 7, 10480. [CrossRef] [PubMed] 